\title{
Correlation between Serum Cystatin C Level and Retinal Blood Flow in Patients with Essential Hypertension
}

\author{
Sumin Huanga Shu Zhang ${ }^{\mathrm{b}}$ Jiajun Wang ${ }^{\mathrm{c}}$ Guanghui Hou ${ }^{\mathrm{a}}$ Shuyi $\mathrm{Xu}^{\mathrm{a}}$ \\ aDepartment of Ophthalmology, Zhuhai People's Hospital (Zhuhai Hospital Affiliated with Jinan University), \\ Zhuhai, PR China; ${ }^{b}$ Department of Neurology, Zhuhai People's Hospital (Zhuhai Hospital Affiliated with Jinan \\ University), Zhuhai, PR China; 'Department of Health Management, Zhuhai People's Hospital (Zhuhai Hospital \\ Affiliated with Jinan University), Zhuhai, PR China
}

\section{Keywords}

Retinal blood flow - Essential hypertension - Macular area ·

Optic disc $\cdot$ Serum cystatin C

\begin{abstract}
Introduction: Hypertension is known to impact the structure and function of the ocular vascular system and is an established risk factor for many eye diseases. This study aimed to detect the blood flow in the optic disc and macula in patients with essential hypertension and to analyze its correlation with serum cystatin C (Cys-C) levels. Methods: This single center, cross-sectional study included 100 patients with primary hypertension without hypertensive retinopathy, who were divided into an experimental group (50 cases, 50 eyes) with elevated serum Cys-C levels and a control group (50 cases, 50 eyes) with normal serum Cys-C. The optic disc and macular vessel density (VD) and vascular perfusion density (PD) were assessed using optical coherence tomography angiography. Data such as the area, perimeter, and circularity of the foveal avascular zone (FAZ) were analyzed. Results: There were statistically significant between-group differences in the VD and PD of the optic disc $(p<0.05)$. Spearman correlation analysis of related indicators revealed that serum
\end{abstract}

Cys-C was positively correlated with creatinine, uric acid, and FAZ circularity $(p<0.05)$. Furthermore, serum Cys-C was negatively correlated with optic disc VD and PD in some regions $(p<0.05)$. Conclusion: In patients with essential hypertension, serum Cys-C is negatively correlated with VD and PD in the inner layer of the optic disc (zone 10).

(C) 2022 The Author(s)

Published by S. Karger AG, Basel

\section{Introduction}

Essential hypertension is the most common chronic cardiovascular disease, with a prevalence of 1.1 billion in 2015. It is estimated that by 2025 , the number of patients suffering from hypertension in the world will increase to 1.5 billion [1]. Hypertension induces long-term effects on the structure and function of the ocular vascular system and is an established risk factor for many eye diseases. Hypertensive retinopathy (HRP) is usually asymptomatic in the early stages. However, during the late stage, it severely impairs vision, reduces the quality of life, and

The first three authors (Sumin Huang MD, Shu Zhang MD, Jiajun Wang MD) are all co-first authors.
(C) 2022 The Author(s)

Published by S. Karger AG, Basel

This is an Open Access article licensed under the Creative Commons Attribution-NonCommercial-4.0 International License (CC BY-NC) (http://www.karger.com/Services/OpenAccessLicense), applicable to the online version of the article only. Usage and distribution for commercial purposes requires written permission.
Correspondence to:

Guanghui Hou, lupia_guiji@163.com

Shuyi Xu, ophthalmology333@163.com 
Table 1. Basic clinical data of the two groups $(\bar{x} \pm S)$

\begin{tabular}{llllr}
\hline & Experimental group & Control group & $t / Z$ & $p$ value \\
\hline Patients (eyes) & 50 & 50 & - & - \\
Gender (male/female) & $21 / 29$ & $19 / 31$ & 0.042 & 0.838 \\
Average age, years & $72.62 \pm 5.97$ & $72.16 \pm 6.81$ & 0.359 & 0.720 \\
Weight, kg & $59.01 \pm 10.81$ & $61.51 \pm 12.82$ & -1.054 & 0.295 \\
Height, cm & $159.64 \pm 7.60$ & $160.04 \pm 9.69$ & -0.230 & 0.819 \\
BMl & $23.08 \pm 3.56$ & $23.85 \pm 3.40$ & -1.113 & 0.269 \\
Disease course, years & $7.06 \pm 5.72$ & $7.46 \pm 6.73$ & -0.320 & 0.749 \\
Hypertension grade (1 grade/2 grade/3 grade) & $13 / 20 / 17$ & $19 / 14 / 17$ & 2.184 & 0.336 \\
Creatinine, $\mu$ mol/L & $85.05 \pm 26.20$ & $64.00 \pm 11.83$ & -4.969 & $<0.001$ \\
Serum Cys-C, mg/L & $1.37 \pm 0.23$ & $0.95 \pm 0.12$ & -8.620 & $<0.001$ \\
Uric acid, $\mu$ mol/L & $372.42 \pm 86.72$ & $324.76 \pm 89.50$ & -2.899 & 0.004 \\
RNFL thickness & $89.46 \pm 13.14$ & $91.94 \pm 12.54$ & -0.828 & 0.408 \\
FAZ area, mm ${ }^{2}$ & $0.22 \pm 0.14$ & $0.19 \pm 0.11$ & -1.042 & 0.298 \\
FAZ perimeter, mm & $1.94 \pm 0.68$ & $1.84 \pm 0.61$ & -0.731 & 0.465 \\
Al & $0.68 \pm 0.10$ & $0.65 \pm 0.12$ & -1.418 & 0.156 \\
\hline
\end{tabular}

BMI, body mass index; RNFL, retinal nerve fiber layer.

increases the economic burden of patients. Therefore, the observation of early retinal changes in patients with hypertension is of considerable importance for the early diagnosis and treatment of HRP.

Evidence has shown that cystatin C (Cys-C) is expressed in vitreous and retinal cells of rats [2]. Retinal pigment epithelial cells are believed to be the main sites of Cys-C secretion [3]. In recent years, studies have explored the relationship between serum Cys-C levels and blood pressure. Peralta et al. [4] observed that systolic blood pressure and serum Cys- $\mathrm{C}$ are positively correlated (for every $1.2 \mathrm{~mm}$ $\mathrm{Hg}$ increase in systolic blood pressure, serum Cys-C raises by $0.4 \mathrm{mg} / \mathrm{L}$ ). A multiethnic study revealed that after adjusting for hypertension risk factors, for every $1 \mathrm{mg} / \mathrm{L}$ increase in serum Cys- $C$, the incidence of hypertension increased by $3.43 \%$ [5]. Therefore, there is a correlation between blood pressure and serum Cys-Clevels. Nevertheless, the relationship between serum Cys- $\mathrm{C}$ and retinal flow density in hypertensive patients is still unclear.

We searched relevant literature and identified the serum Cys-C level as an independent risk factor for diabetic retinopathy (DR). It has been reported that serum Cys-C levels are positively correlated with the severity of DR in patients with type 2 diabetes, demonstrating that when serum Cys-C levels exceed $1.25 \mathrm{mg} / \mathrm{L}$, the risk of severe DR increases 11-fold [6]. Since serum Cys-C levels can predict the development of DR and that HRP and DR are both microvascular diseases, the correlation between serum Cys- $\mathrm{C}$ and retinal blood flow in hypertensive patients warrants further exploration.
We used optical coherence tomography angiography (OCTA) to quantitatively analyze the blood flow of the optic disc and macula, thereby providing objective data at the level of the microvessels to enable the observation of subtle changes over time. This study aimed to detect the optic disc and macular blood flow in patients with essential hypertension using OCTA and to analyze its correlation with Cys-C levels.

\section{Materials and Methods}

\section{Participants}

This cross-sectional study was reviewed and approved by the hospital Ethics Committee and complied with the principles of the Declaration of Helsinki. Written informed consent was obtained from all participants. From June 2020 to July 2021, 100 patients with primary hypertension at Zhuhai People's Hospital were divided into an elevated serum Cys-C (experimental group) (50 cases, 50 eyes; mean age: $72.62 \pm 5.97$ years; men: 21 , women: 29$)$ and a normal serum Cys- $C$ level group (control group) (50 cases, 50 eyes; mean age: $72.16 \pm 6.81$ years; men: 19 , women: 31 ). There was no statistically significant difference in age between the 2 groups $(p>0.05)$. The basic clinical data of the 2 groups are shown in Table 1 .

The inclusion criteria were as follows: patients diagnosed with essential hypertension without HRP, best-corrected visual acuity $\geq 1.0$, intraocular pressure $\leq 21 \mathrm{~mm} \mathrm{Hg}$, equivalent spherical lens between $-1.50 \mathrm{D}$ and $+1.50 \mathrm{D}$, OCTA collection signal $\geq 6$, and three random blood pressure measurements $<140 / 90 \mathrm{~mm} \mathrm{Hg}$ under calm conditions on different recent days. Included in the study were patients with corneal transparency without scar, deep anterior chamber, Tyndall (-), round pupil, iris without front and back adhesion, transparent crystal or only mild turbidity, fundus ex- 
Fig. 1. a Optic disc blood flow density partition (left eye). b Macular area blood flow density partition (right eye).
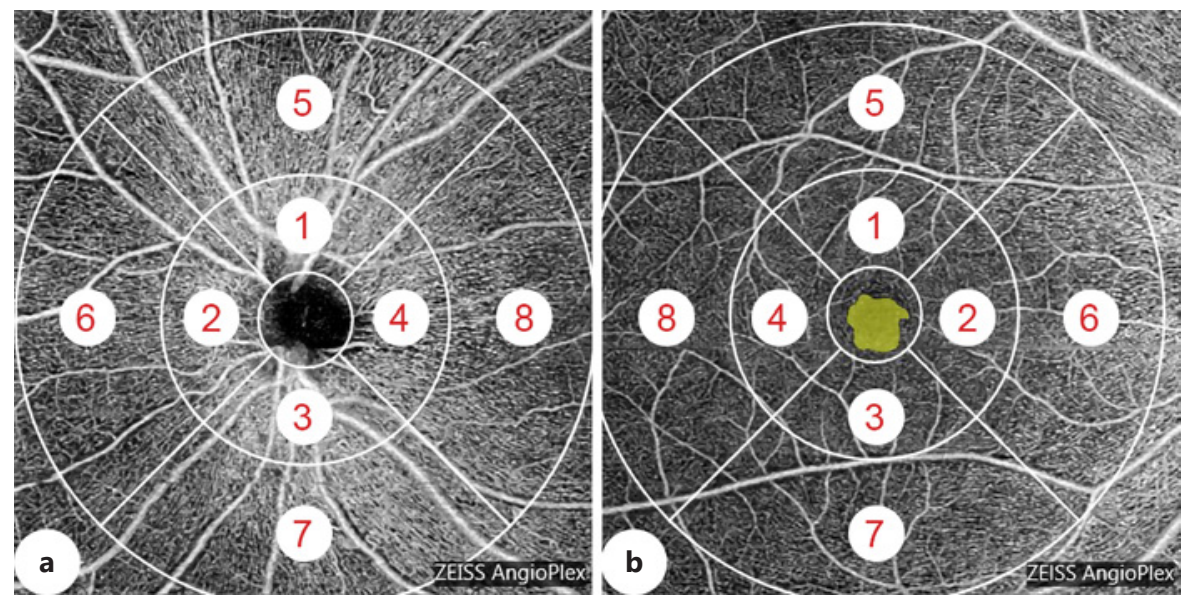

amination of the optic disc is clear, no congestion and edema, C/D $<0.6$, binocular difference $<0.2$, macular morphology and color were normal, and visible reflections underwent OCTA examination.

The exclusion criteria were patients diagnosed with other diseases of the eye, diabetes mellitus, systemic lupus erythematosus, various nephrotic syndromes, tumors, and currently taking drugs that affect serum Cys-C levels, such as glucocorticoids, methotrexate, cyclophosphamide, and tacrolimus capsules.

\section{Methods}

\section{General Examination}

Sex, age, height, weight, body mass index (BMI), course of disease, hypertension grade, blood pressure, computer optometry and apparent refraction, intraocular pressure, fundus photography, slit-lamp examination, and serum $\mathrm{Cys}-\mathrm{C}$ were assessed. All examinations were performed independently by the same physician.

\section{OCTA Examination}

Scanning was performed using Zeiss AngioPlex (CIRRUS HDOCT 5000; Zeiss Meditech Inc., Germany) automatic tracking scan mode to complete horizontal and vertical scanning to obtain a $6 \mathrm{~mm} \times 6 \mathrm{~mm}$ scan image centered on the optic disc and macular area. AngioPlex software will automatically display the measure the superficial retinal capillary plexus (SCP), and the built-in software automatically provides the quantification of SCP. Three concentric circles with diameters of 1,3 , and $6 \mathrm{~mm}$ with the optic disc and the macular area as the center were generated. The two rings outside the optic disc and macular area were equally divided into four regions, respectively, as the upper part of the optic disc and the macular area (defined as areas 1 and 5), the nasal side of the optic disc and the macula (areas 2 and 6), the underside of the optic disc and the macula (area 3 and 7 ), and the temporal side of the optic disc and the macula (areas 4 and 8). We defined the optic disc and the center of the macula as zone 9, areas 1-4 as zone 10 (inner layer), areas 5-8 as zone 11 (outer layer), and areas 1-9 as zone 12 . The vessel density (VD) and vascular perfusion density (PD) were measured in different areas. The VD refers to the total length of the perfusion blood vessel per unit area in the measurement area, and PD defined as the perfusion blood vessel per unit area in the measurement area. The thickness of the retinal nerve fiber layer (RNFL), foveal avascular zone (FAZ) area, FAZ perimeter, and FAZ circularity were assessed. To avoid measurement errors, each subject was scanned three times, and the average value was recorded as the final data for the analysis (Fig. 1).

\section{Statistical Analysis}

Statistical analysis was performed using SPSS version 26.0 (SPSS, Inc., Chicago, IL, USA). The results of descriptive analysis are reported as the mean \pm standard deviation $(x \pm S)$, count data comparisons were made using the $\chi^{2}$ test, measurement data comparisons between groups were tested by homogeneity of variance, and the comparison between groups was via one-way analysis of variance, or a nonparametric rank test was used for nonuniform variance. Spearman rank correlation coefficient was used for correlation analysis. Differences were considered statistically significant at $p<0.05$.

\section{Results}

\section{Basic Clinical Data of the Two Groups}

There were no statistically significant differences in weight, height, BMI, disease course and hypertension grade between the 2 groups $(p>0.05)$. The betweengroup differences in creatinine, uric acid, and serum CysC levels were statistically significant $(p<0.05)$. The basic clinical data of the 2 groups are shown in Table 1.

\section{Optic Disc and Macular Area Blood Flow Density}

There were statistically significant differences in the VD and PD of the optic disc (zones 1, 2, 5, 6, 10, 11, and 12 ) between the 2 groups of patients $(p<0.05)$. There were no statistical differences in RNFL, FAZ area, FAZ perimeter, FAZ circularity, VD and PD of the optic disc 


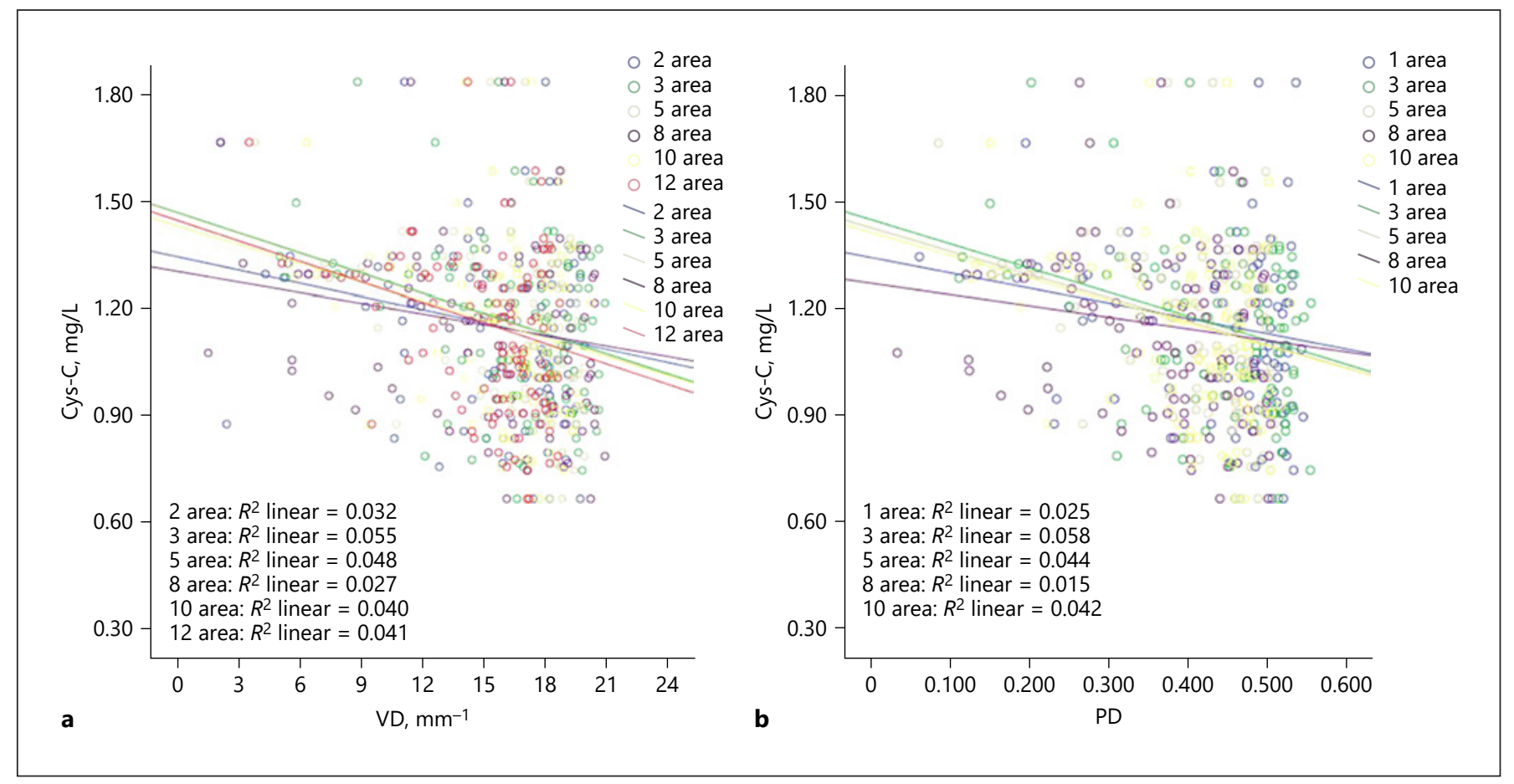

Fig. 2. a Correlation analysis between serum Cys-C and optic disc VD. b Correlation analysis between serum Cys-C and optic disc PD.

Table 2. VD of the optic disc in 2 groups $(\bar{x} \pm S)$

\begin{tabular}{lllll}
\hline & $\begin{array}{l}\text { Experimental } \\
\text { group, } \mathrm{mm}^{-1}\end{array}$ & $\begin{array}{l}\text { Control group, } \\
\mathrm{mm}^{-1}\end{array}$ & $Z$ & $p$ value \\
\hline 1 zone & $16.09 \pm 4.15$ & $18.19 \pm 2.41$ & -2.825 & $\mathbf{0 . 0 0 5}$ \\
2 zone & $14.51 \pm 4.63$ & $16.71 \pm 3.02$ & -2.431 & $\mathbf{0 . 0 1 5}$ \\
3 zone & $15.97 \pm 4.27$ & $17.80 \pm 2.13$ & -1.858 & 0.063 \\
4 zone & $13.23 \pm 5.62$ & $15.47 \pm 4.44$ & -1.875 & 0.061 \\
5 zone & $15.80 \pm 3.86$ & $17.59 \pm 2.13$ & -2.279 & 0.023 \\
6 zone & $13.26 \pm 4.34$ & $15.05 \pm 3.35$ & -2.206 & 0.027 \\
7 zone & $16.05 \pm 3.91$ & $17.31 \pm 2.53$ & -1.276 & 0.202 \\
8 zone & $14.39 \pm 4.78$ & $16.07 \pm 4.29$ & -1.817 & 0.069 \\
9 zone & $4.41 \pm 3.42$ & $4.47 \pm 3.40$ & -0.134 & 0.893 \\
10 zone & $14.97 \pm 3.81$ & $17.01 \pm 1.99$ & -2.820 & 0.005 \\
11 zone & $14.83 \pm 3.84$ & $16.51 \pm 2.35$ & -2.134 & 0.033 \\
12 zone & $14.59 \pm 3.55$ & $16.26 \pm 1.95$ & -2.379 & 0.017 \\
\hline
\end{tabular}

(zones 3, 4, 7, 8, and 9), and VD and PD of various regions of the macula between the 2 groups of patients (Table 1-3).

\section{Correlation Analysis}

Spearman correlation analysis between serum Cys-C and each index revealed that serum Cys- $\mathrm{C}$ was positively correlated with creatinine, uric acid, and FAZ circularity
Table 3. PD of the optic disc in 2 groups $(\bar{x} \pm S)$

\begin{tabular}{lllll}
\hline & $\begin{array}{l}\text { Experimental } \\
\text { group }\end{array}$ & $\begin{array}{l}\text { Control } \\
\text { group }\end{array}$ & $Z$ & $p$ value \\
\hline 1 zone & $0.41 \pm 0.12$ & $0.48 \pm 0.68$ & -2.830 & $\mathbf{0 . 0 0 5}$ \\
2 zone & $0.38 \pm 0.13$ & $0.45 \pm 0.09$ & -2.389 & $\mathbf{0 . 0 1 7}$ \\
3 zone & $0.41 \pm 0.12$ & $0.47 \pm 0.06$ & -1.903 & 0.057 \\
4 zone & $0.32 \pm 0.14$ & $0.37 \pm 0.11$ & -1.737 & 0.082 \\
5 zone & $0.40 \pm 0.11$ & $0.45 \pm 0.06$ & -2.213 & 0.027 \\
6 zone & $0.33 \pm 0.12$ & $0.37 \pm 0.09$ & -1.982 & 0.047 \\
7 zone & $0.40 \pm 0.10$ & $0.44 \pm 0.09$ & -1.400 & 0.162 \\
8 zone & $0.36 \pm 0.10$ & $0.39 \pm 0.11$ & -1.682 & 0.093 \\
9 zone & $0.12 \pm 0.10$ & $0.12 \pm 0.09$ & -0.541 & 0.588 \\
10 zone & $0.38 \pm 0.11$ & $0.44 \pm 0.06$ & -2.858 & $\mathbf{0 . 0 0 4}$ \\
11 zone & $0.37 \pm 0.10$ & $0.41 \pm 0.06$ & -2.003 & $\mathbf{0 . 0 4 5}$ \\
12 zone & $0.37 \pm 0.11$ & $0.41 \pm 0.05$ & -1.999 & $\mathbf{0 . 0 4 6}$ \\
\hline
\end{tabular}

$(p<0.05)$. Furthermore, it was negatively correlated with the optic disc VD (zones 2, 3, 5, 8, 10, and 12) and optic disc PD (zones 1, 3, 5, 8, and 10) $(p<0.05)$. Serum Cys-C was not correlated with age, weight, height, BMI, disease course, RNFL thickness, FAZ area, FAZ perimeter, optic disc VD (zones 1, 4, 6, 7, 9, and 11), optic disc PD (zones $2,4,6,7,9,11$, and 12), and macular zone VD and PD $(p>0.05)$ as shown in Figure 2. 


\section{Discussion}

This study used OCTA to quantify the retinal blood flow in patients with essential hypertension with various serum Cys-C levels. In patients with elevated serum Cys$\mathrm{C}$ levels and well-controlled blood pressure, without HRP, the superficial layers of the VD and PD were lower than those of patients with normal serum Cys-C levels. This suggests that retinal hypoperfusion exists regardless of hypertensive retinal complications. Serum Cys-C was negatively correlated with VD and PD in the inner layer of the optic disc (zone 10). The higher the serum Cys-C level, the lower the blood flow of the optic disc (inner layer).

Cys- $\mathrm{C}$ is a nonglycosylated cysteine protease inhibitor. Its rate of production and blood concentration are stable; are hardly affected by age, sex, inflammation, and physiological factors $[7,8]$; and are mainly distributed in tissue nucleated cells and body fluids. There is evidence that Cys- $\mathrm{C}$ is expressed in the vitreous and various layers of retinal cells of the rat [2] and that the retinal pigment epithelial cells are the main secretion sites of Cys-C [3]. There is an association between the blood pressure level and serum Cys-C. However, there is currently no research on Cys-C and retinal blood flow in patients with hypertension.

Hypertension induces long-term effects on the structure and function of the ocular vascular system. The morphological and physiological characteristics of the microvessels of the retina can be used as prospective indicators to evaluate retinal microvascular diseases and monitor end-organ damage related to hypertension [9, 10]. Traditional fundus fluorescein angiography is the gold standard for the diagnosis of fundus vascular diseases; however, there are limitations, such as unquantifiable images and invasiveness. In recent years, OCTA underwent rapid development. Advantages of OCTA include that it is noninvasive, rapid, and quantitative. It possesses the function of structural OCT to detect the morphology and structure of the retina and can also directly observe the growth of nonperfusion capillary areas and pathological blood vessels, which are early detection lesions. Tracking of changes in the condition provides objective evidence. A literature analysis suggests that the use of OCTA can improve the predictive value of retinal diseases $[11,12]$. Therefore, OCTA may be the preferred method for monitoring early retinal microvascular changes and predicting end-organ damage in patients with hypertension. In the past, most of the literature regarding the use of OCTA to quantify the retinal VD in patients with hypertension was based on PD. However, there are few studies on PD combined with VD evaluation indicators. To facilitate a more detailed understanding of the microvascular characteristics of hypertensive patients, this study combines VD and PD and divides the entire ring into four quadrants and three ring regions to determine whether there are regional differences and to more comprehensively display the fundus of hypertensive patients.

Our results revealed that the higher the serum Cys-C level, the higher the creatinine and uric acid levels. Serum Cys-C can freely pass through the glomerulus without being secreted by the renal tubules [13]. Gompou et al. [14] reported that mild changes in the estimated glomerular filtration rate (eGFR) caused an increase in serum Cys-C, whereas serum creatinine increased only when the eGFR decreased more significantly. The renal toxicity of hyperuric acid can lead to glomerular damage and a decrease in the eGFR. Therefore, creatinine, hyperuric acid, and serum Cys- $C$ act via the same mechanism.

We also observed that the VD and PD of the nasal, upper, inner, outer, and entire optic discs of the experimental group were lower than those of the control group. The VD and PD of the temporal and the underside optic discs of the experimental group were not lower than those of the control group. There are no previous studies combining serum Cys-C and retinal blood flow density in patients with hypertension. Important aspects of this study are as follows: (1) this study demonstrates that even in patients with well-controlled hypertension and nonHRP, OCTA can detect changes in the superficial retinal capillaries. OCTA can be used as a primary examination method for diagnosing fundus vascular diseases; (2) VD and PD in the nasal side and upper part of the optic disc in the experimental group were statistically different from those in the control group. A previous study [15] reported that RNFL thickness is positively correlated with the density of the microvessels near the optic disc, with the thinnest RNFL on the nasal side and the least blood flow being the nasal blood flow. Those results are in agreement with the results of our study, which revealed a decrease in nasal blood flow in the experimental group compared with that of the control group. Gangwani Rita et al. [16] highlighted that mean arterial pressure is negatively correlated with the retinal blood flow above the optic disc and on the nasal side, which is consistent with the results of this study; (3) this study shows that the temporal side of the optic disc in the experimental group and the VD and PD below are not statistically different from those in the control group. We believe that this is due to the exis- 
tence of the papillary macular tract in this area; therefore, it is not susceptible to hypertension and the influence of the increased serum Cys-C level; and (4) we also observed that the VD and PD of the inner, outer, and entire optic discs of the experimental group were lower than those of the control group. Serum Cys-C can increase the release of various inflammatory factors and mediate inflammation [17]. We speculate that high levels of Cys-C mediate inflammatory reactions, increase oxygen consumption and blood flow in the fundus retina, increase the release of nitric oxide from vascular endothelial cells, and also disrupt the secretion of vascular endothelin-1, thereby causing vascular endothelial function disorder or direct damage to the endothelial cells, leading to vasomotor dysfunction, resulting in a decrease in retinal blood flow. From the mechanism of action, HRP is closely related to vascular endothelial regulation and inflammatory factors [18]. Serum Cys-C may be involved in pathological changes such as arterial wall remodeling, vascular integrity, neovascularization, inflammation, and neuronal degeneration [19]. Therefore, we believe that the common pathway of serum Cys-C and HRP can explain the close connection between the two.

This study revealed that serum Cys-C has a correlation with the optic disc area, but it is not regional. It is certain that the VD and PD in the inner layer of the optic disc (zone 10) were negatively correlated with the serum Cys$\mathrm{C}$ level. However, the data of the macular area have no correlation with serum Cys-C. We postulate that the above results may be related to the fact that the central retinal artery is branched from the ophthalmic artery branch of the carotid artery, passing through the optic disc before traveling to various quadrants of the retina. The blood flow of the layer (zone 10) will be preferentially affected by the systemic blood pressure fluctuations and increased serum Cys- $\mathrm{C}$ levels. Cherry spots occur when the central retinal artery is blocked, In addition to the retinal circulatory system in the macular area, there is also a rich choroidal circulatory system that supplies; therefore, it is less affected by high blood pressure and increased Cys-C levels. Peng et al. [20] pointed out that there was no significant difference in macular vascular density parameters among different HRP grades, which was consistent with the results of this study. The difference is that studies have shown that severe hypertension will also affect the blood flow density in the macular area over time [21]. This may also be related to HRP patients who were not included this study because the studies [22] have pointed out that kidney damage is correlated with HRP grading, and it was found that compared with the patients with I HRP, patients with II and III HRP were 3.9 and 5.6 times more likely to have renal impairment, respectively.

This study has certain limitations. First, this was a retrospective, cross-sectional study. The actual duration of hypertension may be longer than the time the patient was diagnosed with hypertension. Second, antihypertensive drugs and kidney function could affect retinal microcirculation $[23,24]$. Third, we only analyzed SCP and excluded the deep retinal capillary plexus and choroidal capillary blood flow. The reason is that with the scanning of Zeiss AngioPlex, projection artifacts appeared in most of the deep retinal images, affecting the quantitative analysis of deep retinal capillary plexus and choroidal capillaries. Christian et al. [25] reported that $75 \%$ of deep retinal images showed projection artifacts.

The novelty of this study lies in further exploration of the correlation between serum Cys-C levels and retinal blood flow based on the use of OCTA. The results showed that the increased serum Cys-C level can predict decreased blood flow density in the optic disc (inner layer). Therefore, based on this research, whether OCTA can be used for early diagnosis of HRP in the future can be considered and analyze the morphological characteristics of retinal microvascular changes in HRP patients, including capillary sparseness, microhemangioma, macular pedicle ring defect, and no capillary perfusion. The traditional HRP diagnosis and grading method is based on fundus photography images and then referred to the Keith-Wagener-Barker grading evaluation criteria to grade HRP. It is a subjective classification method with certain limitations.

\section{Conclusion}

In patients with essential hypertension, serum Cys- $\mathrm{C}$ is negatively correlated with VD and PD in the inner layer of the optic disc (zone 10). The serum Cys-C level should be an indicator of concern that affects retinal blood flow in patients with essential hypertension. The blood flow in the optic disc (inner layer) can be used as a key observation area for patients with elevated serum Cys-C.

\section{Statement of Ethics}

The study has been granted an exemption from requiring ethics approval of Zhuhai People's hospital Ethics Committee. Written informed consent was obtained from all participants. 


\section{Conflict of Interest Statement}

The authors declared no potential conflicts of interest with respect to the research, authorship, and/or publication of this article.

\section{Funding Sources}

This study was supported by grants from the Science and Technology Foundation of Zhuhai (ZH3310200025PJL) and the Cultivation Project of Zhuhai People's Hospital (2019PY-22).

\section{Author Contributions}

All the authors contributed equally to the study.

\section{Data Availability Statement}

All data generated or analyzed during this study are included in this article. Further inquiries can be directed to the corresponding author.

\section{References}

1 Forouzanfar MH, Liu P, Roth GA, Ng M, Biryukov S, Marczak L, et al. Global burden of hypertension and systolic blood pressure of at least 110-115 mm Hg, 1990-2015. JAMA. 2017;317(2):165-82.

2 Ahuja S, Ahuja-Jensen P, Johnson LE, Caffé AR, Abrahamson M, Ekström PA, et al. rd1 mouse retina shows an imbalance in the activity of cysteine protease cathepsins and their endogenous inhibitor cystatin C. Invest Ophthalmol Vis Sci. 2008;49(3):1089-96.

3 Luminita P, Paul H, Christine G, Ian G. Cystatin $\mathrm{C}$ in macular and neuronal degenerations: implications for mechanism(s) of agerelated macular degeneration. Vis Res. 2010; 50(7):737-42.

4 Peralta CA, Whooley MA, JoachimIx H, Shlipak MG. Kidney function and systolic blood pressure new insights from cystatin C: data from the Heart and Soul Study. Am J Hypertens. 2006;19(9):939-46.

5 Karine S, Klein Barbara EK, Lee Kristine E, Tsai Michael Y, Klein R. Serum cystatin C and the incidence of hypertension in type 1 diabetes mellitus. Am J Hypertens. 2011;24(1):5963.

6 He R, Shen J, Zhao J, Zeng H, Li L, Zhao J, et al. High cystatin $\mathrm{C}$ levels predict severe retinopathy in type 2 diabetes patients. Eur J Epidemiol. 2013;28(9):775-8.

7 Go YM, Jones DP. Cysteine/cystine redox signaling in cardiovascular disease. Free Radic Biol Med. 2011;50(4):495-509.

8 Yeung BHY, Wong KY, Lin MC, Wong CK, Mashima T, Tsuruo T, et al. Chemosensitisation by manganese superoxide dismutase inhibition is caspase-9 dependent and involves extracellular signal-regulated kinase $1 / 2 . \mathrm{Br} J$ Cancer. 2008;99(2):283-93.

9 Wong TY, Klein R, Sharrett AR, Duncan BB, Couper DJ, Tielsch JM, et al. Retinal arteriolar narrowing and risk of coronary heart disease in men and women: the Atherosclerosis Risk in Communities Study. JAMA. 2002;287(9): 1153-9.

10 Patton N, Aslam T, MacGillivray T, Pattie A, Deary IJ, Dhillon B. Retinal vascular image analysis as a potential screening tool for cerebrovascular disease: a rationale based on homology between cerebral and retinal microvasculatures. J Anat. 2005;206(4):319-48.

11 Wang J, Jiang J, Zhang Y, Qian YW, Zhang JF, Wang ZL. Retinal and choroidal vascular changes in coronary heart disease: an optical coherence tomography angiography study. Biomed Opt Express. 2019;10(4):1532-44.

12 Alan G, Guenancia C, Arnould L, Azemar A, Pitois S, Maza M, et al. Retinal vascular density as a novel biomarker of acute renal injury after acute coronary syndrome. Sci Rep. 2019; 9(4):8060.

13 Rigalleau V, Beauvieux MC, Le Moigne F, Lasseur C, Chauveau P, Raffaitin C, et al. Cystatin $\mathrm{C}$ improves the diagnosis and stratification of chronic kidney disease, and the estimation of glomerular filtration rate in diabetes. Diabetes Metabol. 2008;34(5):482-9.

14 Gompou A, Perrea D, Karatzas T, Bellos JK, Kastania AN, Boletis I, et al. Relationship of changes in cystatin-c with serum creatinine and estimated glomerular filtration rate in kidney transplantation. Transplant Proc. 2015;47(6):1662-74.

15 Lin Y. Quantitative analysis of peripapillary and macular vessel densities in primary hypertensive patients. Fujian Medical University; 2018.

16 Gangwani Rita A, Lee Jacky WY, Mo HY, Sum R, Kwong Alfred SK, Wang Jenny HL, et al. The correlation of retinal nerve fiber layer thickness with blood pressure in a Chinese hypertensive population. Medicine. 2015; 94(23):e947.

17 Yang H, Wang C, Guo M, Zhou Y, Feng Z, Yin Z. Correlations between peroxisome proliferator activator receptor $\gamma$, cystatin $\mathrm{C}$, or advanced oxidation protein product, and atherosclerosis in diabetes patients. Pathol Res Pract. 2015;211(3):235-9.

18 Han Y, Jiao R, Zhang A, Cui Y, Zhang C. Relationships between levels of miroRNA 124 and miroRNA 143 in peripheral blood and hypertensive retinopathy. Chin J Difficult Complicated Cases. 2020;19(3):275-9.

19 Mussap M, Mario P. Biochemistry and clinical role of human cystatin C. Crit Rev Clin Lab Sci. 2004;41(5-6):467-550.

20 Peng Q, Hu Y, Huang M, Wu Y, Zhong P, Dong $X$, et al. Retinal neurovascular impairment in patients with essential hypertension: an Optical Coherence Tomography Angiography Study. Invest Ophthalmol Vis Sci. 2020;61(8):42.

21 Han-Min L, Woo-Hyuk L, KimNam K, Young Joon J, Jung KY. Changes in thickness of central macula and retinal nerve fibre layer in severe hypertensive retinopathy: a 1-year longitudinal study. Acta Ophthalmol. 2018; 96(3):e386-92.

22 Liu Y, Li J, Pan J, Wang Y, Mao G, Jiang Z. Morphological changes in and quantitative analysis of macular retinal microvasculature by optical coherence tomography angiography in hypertensive retinopathy. Hypertens Res. 2021;44(3):325-36.

23 Hughes AD, AliceStanton V, AtifJabbar S, Chapman N, Elena Martinez-Perez M, Thom SAMG. Effect of antihypertensive treatment on retinal microvascular changes in hypertension. J Hypertens. 2008;26(8):1703-7.

24 Bosch A, Scheppach Johannes B, Harazny Joanna M, Ulrike R, Kai-Uwe E, Schmieder Roland E, et al. Retinal capillary and arteriolar changes in patients with chronic kidney disease. Microvasc Res. 2018;118:121-7.

25 Christian E, Lang Gabriele E, Jens D, Max L, Lang Gerhard K, Werner Jens U. Quantity and quality of image artifacts in optical coherence tomography angiography. PLoS One. 2019;14(1):e0210505.
Cystatin C and Retinal Blood Flow in Hypertension
Ophthalmic Res 2022;65:335-341 DOI: $10.1159 / 000522219$ 